\title{
Some new generalized weighted dynamic inequalities of Hardy's type on time scales
}

\author{
S. H. Saker ${ }^{a}$, M. M. A. El-sheikh ${ }^{b}$, A. M. Madian ${ }^{b, *}$
}

${ }^{a}$ Department of Mathematics, Faculty of Science, Mansoura University, Mansoura, Egypt.

${ }^{b}$ Department of Mathematics, Faculty of Science, Menoufia University, Menoufia, Egypt.

\begin{abstract}
In this paper, we will prove some new generalized weighted dynamic inequalities of Hardy's type on a time scale $\mathbb{T}$. The obtained results contain as special cases some published results when the time scale $\mathbb{T}=\mathbb{R}$ and when $\mathbb{T}=\mathbb{N}$. The results, to the best of the authors' knowledge, are essentially new.
\end{abstract}

Keywords: Hardy type inequality, monotonic functions, time scales, dynamic inequalities.

2020 MSC: 26D10, 26D15, 34N05, 47B38, 39A12.

(C)2021 All rights reserved.

\section{Introduction}

In 1920, Hardy [22] proved that if $p>1$, and $a_{n} \geqslant 0$ for $n \geqslant 1$, then

$$
\sum_{n=1}^{\infty}\left(\frac{1}{n} \sum_{k=1}^{n} a_{k}\right)^{p} \leqslant\left(\frac{p}{p-1}\right)^{p} \sum_{n=1}^{\infty} a_{n}^{p}, \quad p>1 .
$$

In 1925, Hardy [23] proved the continuous version of the inequality (1.1) via calculus of variations, which states that: If $p>1, f \geqslant 0$ over interval $(0, \infty)$ and $\int_{0}^{\infty} f^{p}(x) d x<\infty$, then

$$
\int_{0}^{\infty}\left(\frac{1}{x} \int_{0}^{x} f(t) d t\right)^{p} d x \leqslant\left(\frac{p}{p-1}\right)^{p} \int_{0}^{\infty} f^{p}(x) d x
$$

The constant $(p /(p-1))^{p}$ in (1.1) and (1.2) is the best possible. Also in 1925 [23, Theorem B] Hardy generalized (1.1) and proved that if $p>1, f(n) \geqslant 0, w(n)>0$ and $\Lambda(n)=\sum_{k=1}^{n} w(k)$, then

$$
\sum_{n=1}^{\infty} w(n)\left(\frac{1}{\Lambda(n)} \sum_{k=1}^{n} w(k) f(k)\right)^{p} \leqslant\left(\frac{p}{p-1}\right)^{p} \sum_{n=1}^{\infty} w(n) f^{p}(n) .
$$

\footnotetext{
*Corresponding author

Email address: Aisha.mahmoud5993@gmail.com (A. M. Madian)

doi: 10.22436/jmcs.023.04.02
}

Received: 2020-08-12 Revised: 2020-09-01 Accepted: 2020-10-03 
For more generalizations, extensions and applications of these inequalities, we refer the reader to the papers [8-10, 18, 26, 28, 32, 33] and the books [24, 25]. In 1928, Copson [19] generalized (1.3) and proved that if $p \geqslant c>1, f(n) \geqslant 0$ and $w(n)>0$ for $n \geqslant 1$, then

$$
\sum_{n=1}^{\infty} \frac{w(n)}{\Lambda^{c}(n)}\left(\sum_{k=1}^{n} w(k) f(k)\right)^{p} \leqslant\left(\frac{p}{c-1}\right)^{p} \sum_{n=1}^{\infty} w(n) \Lambda^{p-c}(n) f^{p}(n) .
$$

In 1976, Copson [20] established the continuous version of (1.4) and proved that if $p \geqslant 1, c>1$, then

$$
\int_{0}^{b} \frac{w(x) F^{p}(x)}{\Lambda^{c}(x)} d x \leqslant\left(\frac{p}{c-1}\right)^{p} \int_{0}^{b} w(x) \Lambda^{p-c}(x) f^{p}(x) d x
$$

where

$$
\Lambda(x)=\int_{0}^{x} w(t) d t, \quad \text { and } \quad F(x)=\int_{0}^{x} w(t) f(t) d t
$$

In 1990, Mohapatra and Vajravelu [27] proved new extensions of Copson's type inequality (1.5). In particular, they proved that if $w(x)$ is positive and continuous in $[0, \infty)$ and there exist positive constants $A$ and $B$ such that

$$
x\left|w^{\prime}(x)\right| \leqslant A w(x), \text { and } x w(x) \leqslant B \wedge(x), \text { for all } x>0
$$

then

$$
\int_{0}^{\infty} w(x)\left(\frac{F(x)}{\Lambda(x)}\right)^{p} d x \leqslant(A+B)^{p} \int_{0}^{\infty}\left(\frac{1}{x} \int_{0}^{x} w^{\frac{1}{p}}(t) f(t) d t\right)^{p} d x
$$

for $f(x) \geqslant 0$ and $p>1$. Also, in [27] they proved that if (1.6) holds and $w^{1 / p}(t) f(t) / t \in L^{1}(x, \infty)$ for all $x>0$, then

$$
\int_{0}^{\infty} w(x)\left(\int_{x}^{\infty} \frac{w(t) f(t)}{\Lambda(t)} d t\right)^{p} d x \leqslant(B+p C)^{p} \int_{0}^{\infty}\left(\int_{x}^{\infty} \frac{w^{\frac{1}{p}}(t) f(t)}{t} d t\right)^{p} d x
$$

where $C=1+A+B$.

In the last decades some authors have been interested in finding some discrete results on $l^{p}(\mathbb{N})$ analogues to $L^{p}(\mathbb{R})$-bounds in different fields in analysis and as a result this subject becomes a topic of ongoing researches. One reason for this upsurge of interest in discrete case is also due to the fact that discrete operators may even behave differently from their continuous counterparts (see [17, 36]). So it is natural to look for the discrete versions of the inequalities (1.7) and (1.8) which is one of our aims. On the other hand, the study of dynamic inequalities on time scales has received a lot of attention in recent years and becomes a major field in pure and applied mathematics. The time scale $\mathbb{T}$ is an arbitrary nonempty closed subset of the real numbers $\mathbb{R}$. These results contain the classical continuous and discrete inequalities as special cases when $\mathbb{T}=\mathbb{R}$ and $\mathbb{T}=\mathbb{N}$ and can be extended to different inequalities on different time scales such as $\mathbb{T}=h \mathbb{N}, h>0, \mathbb{T}=q^{\mathbb{N}}$ for $q>1$, etc. For more details about the dynamic inequalities on time scales, we refer the reader to the books $[2,3,13]$ and the papers $[1,6,7,10,11,35]$.

However a lot of results have been written for dynamic inequalities on time scales, there are few results have been written for the weighted dynamic inequalities of Hardy's type on time scales. So, we confine ourselves in this paper in proving some new weighted inequalities on a time scale $\mathbb{T}$, which contain the discrete versions of inequalities of (1.7) and (1.8) as special cases. For completeness, in the following, we present some related results of dynamic inequalities on time scales. In [29] Saker proved that if $p>1$ and $\lambda, g \in C_{r d}\left([a, \infty)_{\mathbb{T}}, \mathbb{R}^{+}\right)$, then

$$
\int_{a}^{\infty} \lambda(t)\left(\Phi^{\sigma}(t)\right)^{p} \Delta t \leqslant p^{p} \int_{a}^{\infty} \lambda^{1-p}(t) \Lambda^{p}(t) g^{p}(t) \Delta t
$$

where

$$
\Lambda(t)=\int_{t}^{\infty} \lambda(s) \Delta s, \text { and } \Phi(t)=\int_{a}^{t} g(s) \Delta s, \text { for } t \in[a, \infty)_{\mathbb{T}}
$$


In [30] Saker, O'Regan and Agarwal proved that if $a \in[0, \infty)_{\mathbb{T}}, p>1$ and $\lambda, g \in C_{r_{d}}\left([a, \infty)_{\mathbb{T}}, \mathbb{R}^{+}\right)$, then

$$
\int_{a}^{\infty} \lambda(t) \Phi^{p}(t) \Delta t \leqslant p^{p} \int_{a}^{\infty} \lambda(t) g^{p}(t) \Delta t
$$

where

$$
\Lambda(t)=\int_{a}^{t} \lambda(s) \Delta s, \text { and } \Phi(t)=\int_{t}^{\infty} \frac{\lambda(s) g(s)}{\Lambda^{\sigma}(s)} \Delta s, \text { for } t \in[a, \infty)_{\mathbb{T}} .
$$

Also, the authors [30] proved that if $a \in[0, \infty)_{\mathbb{T}}$ and $p>1$, then

$$
\int_{a}^{\infty} \lambda(t) \Phi^{p}(t) \Delta t \leqslant p^{p} \int_{a}^{\infty} \frac{\Lambda^{\sigma}(t)}{\Lambda(t)} \lambda(t) g^{p}(t) \Delta t
$$

where

$$
\Lambda(t)=\int_{a}^{t} \lambda(s) \Delta s \text { and } \Phi(t)=\int_{t}^{\infty} \frac{\lambda(s) g(s)}{\Lambda(s)} \Delta s, \text { for } t \in[a, \infty)_{\mathbb{T}} .
$$

Also in [30] they proved that if $a \in[0, \infty)_{\mathbb{T}}, p>1$ and $\lambda(s)>0, f(s) \geqslant 0 \forall s \in[a, \infty)_{\mathbb{T}}$, then

$$
\int_{a}^{\infty} \frac{\lambda(t)}{\left(\Lambda^{\sigma}(t)\right)^{p}}\left(\Psi^{\sigma}(t)\right)^{p} \Delta t \leqslant\left(\frac{p}{p-1}\right)^{p} \int_{a}^{\infty}\left(\frac{\Lambda^{\sigma}(t)}{\Lambda(t)}\right)^{p(p-1)} \lambda(t) f^{p}(t) \Delta t,
$$

where

$$
\Psi(t)=\int_{a}^{t} \lambda(s) f(s) \Delta s \text { and } \Lambda(t)=\int_{a}^{t} \lambda(s) \Delta s \text {, for } t \in[a, \infty)_{\mathbb{T}},
$$

with $\Lambda(\infty)=\infty$. In [31] Saker, O'Regan and Agarwal proved that if $a \in[0, \infty)_{\mathbb{T}}, p \geqslant c>1$ and $\lambda$, $\mathrm{g} \in \mathrm{C}_{\mathrm{rd}}\left([\mathrm{a}, \infty)_{\mathbb{T}}, \mathbb{R}^{+}\right)$, then

$$
\int_{a}^{\infty} \lambda(t) \frac{\left(\Phi^{\sigma}(t)\right)^{p}}{\left(\Lambda^{\sigma}(t)\right)^{c}} \Delta t \leqslant\left(\frac{p}{c-1}\right)^{p} \int_{a}^{\infty} \frac{\left(\Lambda^{\sigma}(t)\right)^{(p-1) c}}{(\Lambda(t))^{p(c-1)}} \lambda(t) g^{p}(t) \Delta t,
$$

where

$$
\Lambda(t)=\int_{a}^{t} \lambda(s) \Delta s, \quad \text { and } \Phi(t)=\int_{a}^{t} \lambda(s) g(s) \Delta s, \text { for } t \in[a, \infty)_{\mathbb{T}} .
$$

Also, the authors [31] proved that if $a \in[0, \infty)_{\mathbb{T}}, p>1,0 \leqslant c<1$ and $\lambda, g \in C_{r d}\left([a, \infty)_{\mathbb{T}}, \mathbb{R}^{+}\right)$, then

$$
\int_{a}^{\infty} \lambda(t) \frac{(\bar{\Phi}(t))^{p}}{\left(\Lambda^{\sigma}(t)\right)^{c}} \Delta t \leqslant\left(\frac{p}{1-c}\right)^{p} \int_{a}^{\infty}\left(\Lambda^{\sigma}(t)\right)^{p-c} \lambda(t) g^{p}(t) \Delta t,
$$

where

$$
\Lambda(t)=\int_{a}^{t} \lambda(s) \Delta s \text { and } \bar{\Phi}(t)=\int_{t}^{\infty} \lambda(s) g(s) \Delta s \text {, for } t \in[a, \infty)_{\mathbb{T}} .
$$

In [33] Saker et al. proved that if $a \in[0, \infty)_{\mathbb{T}}, 1<\gamma \leqslant p$ and $\lambda, q, f \in C_{r d}\left([a, \infty)_{\mathbb{T}}, \mathbb{R}^{+}\right)$such that $q(t)$ is an increasing function on $[a, \infty)_{\mathbb{T}}$, furthermore, if there exists a constant $K>0$ such that

$$
\gamma-1+\frac{q^{\Delta}(t) \Lambda^{\sigma}(t) \Phi^{p}(t)}{\lambda(t)\left[q^{\sigma}(t)\right]^{2}\left(\Phi^{\sigma}(t)\right)^{p}} \geqslant \frac{p}{K^{\prime}}, \text { for } t \in[a, \infty)_{\mathbb{T}}
$$

then

$$
\int_{a}^{\infty} \frac{\lambda(t)}{\left(\Lambda^{\sigma}(t)\right)^{\gamma}}\left(\Phi^{\sigma}(t)\right)^{p} \Delta t \leqslant K^{p} \int_{a}^{\infty} \frac{\left(\Lambda^{\sigma}(t)\right)^{\gamma(p-1)}}{(\Lambda(t))^{(\gamma-1) p}} \lambda(t) f^{p}(t) \Delta t
$$

where

$$
\Lambda(t)=\int_{a}^{t} \lambda(s) q^{\sigma}(s) \Delta s \text { and } \Phi(t)=\int_{a}^{t} \lambda(s) q(s) f(s) \Delta s, \text { for } t \in[a, \infty)_{\mathbb{T}}
$$


Also, the authors in [33] proved that if $a \in[0, \infty)_{\mathbb{T}}, p>1,0 \leqslant \gamma<1$ and $q(t)$ is an increasing function on $[a, \infty)_{\mathbb{T}}$, furthermore, if there exists a constant $K>0$ such that

$$
1-\gamma-\frac{q^{\Delta}(t) \wedge^{\sigma}(t)}{\lambda(t) q^{2}(t)} \geqslant \frac{p}{K}, \text { for } t \in[a, \infty)_{\mathbb{T}}
$$

then

$$
\int_{a}^{\infty} \frac{\lambda(t)}{\left(\Lambda^{\sigma}(t)\right)^{\gamma}}(\bar{\Phi}(t))^{p} \Delta t \leqslant k^{p} \int_{a}^{\infty}\left(\Lambda^{\sigma}(t)\right)^{p-\gamma} \lambda(t) f^{p}(t) \Delta t
$$

where $\Lambda(t)=\int_{a}^{t} \lambda(s) q(s) \Delta s$ and $\bar{\Phi}(t)=\int_{t}^{\infty} \lambda(s) q(s) f(s) \Delta s$, for $t \in[a, \infty)_{\mathbb{T}}$.

Following this trend and to develop the study of dynamic inequalities, we will prove some new weighted dynamic inequalities of Hardy's type on time scales. Our results when $\mathbb{T}=\mathbb{R}$ give the characterizations of the inequalities (1.7) and (1.8) proved by Mohapatra and Vajravelu [27] and when $\mathbb{T}=\mathbb{N}$ our results are essentially new. The paper is organized as follows. In Section 2, we present some preliminaries concerning the theory of time scales and present the basic lemmas that will needed to prove the main results. In Section 3, we prove the main results and derive the discrete versions of the inequalities (1.7) and (1.8) which, to the best of the authors' knowledge are essentially new.

\section{Preliminaries and basic lemmas}

In this section, we recall the following concepts related to the notions of time scales. For more details of time scale analysis, we refer the reader to the two books by Bohner and Peterson $[12,13]$ which summarize and organize much of the time scale calculus. A time scale $\mathbb{T}$ is an arbitrary nonempty closed subset of the real numbers $\mathbb{R}$. We define the time scale interval $[a, b]_{\mathbb{T}}$ by $[a, b]_{\mathbb{T}}:=[a, b] \cap \mathbb{T}$.

Definition 2.1. Let $\mathbb{T}$ be a time scale. For $t \in \mathbb{T}$, we define the forward jump operator $\sigma: \mathbb{T} \rightarrow \mathbb{T}$ by

$$
\sigma(t):=\inf \{s \in \mathbb{T}: s>t\},
$$

while the backward jump operator $\rho: \mathbb{T} \rightarrow \mathbb{T}$ is defined by

$$
\rho(t):=\sup \{s \in \mathbb{T}: s>t\} .
$$

A point $t \in \mathbb{T}$ is said to be right-dense if $\sigma(t):=t$, right-scattered if $\sigma(t)>t$, left-dense if $\rho(t):=t$ and is left-scattered if $\rho(t)<t$. The points that are right-scattered and left-scattered at the same time are called isolated and the points that are simultaneously right-dense and left-dense are called dense. The graininess $\mu$ for on time scale $\mathbb{T}$ is defined by $\mu(t):=\sigma(t)-t$, and for a function $f: \mathbb{T} \rightarrow \mathbb{R}$ the notation $f^{\sigma}(t)$ denotes $f(\sigma(t))$.

A function $f: \mathbb{T} \rightarrow \mathbb{R}$ is said to be right-dense continuous (rd-continuous) provided $f$ is right continuous at right dense points and there exists a finite left limit at all left-dense points. The set of all such rd-continuous functions is denoted by $C_{r d}(\mathbb{T})=C_{r d}(\mathbb{T}, \mathbb{R})$. We assume throughout that $\mathbb{T}$ has the topology that it inherits from the standard topology on the real numbers $\mathbb{R}$.

In this paper, we will refer to the Cauchy (delta) integral of $g$ is defined by $\int_{a}^{t} g(x) \Delta x:=G(t)-G(a)$. It can be shown (see [15]) that if $g \in C_{r d}(\mathbb{T})$, then the Cauchy integral $G(t):=\int_{t_{0}}^{t} g(x) \Delta x$ exists, $t_{0} \in \mathbb{T}$, and satisfies $G^{\Delta}(t)=g(t), t \in \mathbb{T}$. An infinite integral is defined as $\int_{a}^{\infty} f(x) \Delta x:=\lim _{b \rightarrow \infty} \int_{a}^{b} f(x) \Delta x$. In case $\mathbb{T}=\mathbb{R}$, we have $\sigma(t)=\rho(t)=t, \mu(t)=0, f^{\Delta}=f^{\prime}$, and

$$
\int_{a}^{b} f(t) \Delta t=\int_{a}^{b} f(t) d t
$$

and in case $\mathbb{T}=\mathbb{Z}$, we have $\sigma(t)=t+1, \rho(t)=t-1, \mu(t)=1, f^{\Delta}=\Delta f$, and

$$
\int_{a}^{b} f(t) \Delta t=\sum_{t=a}^{b-1} f(t) .
$$


The product and quotient rules for the derivative of the product $f g$ and the quotient $f / g$ (where $g g^{\sigma} \neq 0$, here $g^{\sigma}=g \circ \sigma$ ) of two delta differentiable functions $f$ and $g$ are

$$
\left\{\begin{array}{l}
(\mathrm{fg})^{\Delta}=f g^{\Delta}+\mathrm{f}^{\Delta} \mathrm{g}^{\sigma}=\mathrm{f}^{\Delta} \mathrm{g}+\mathrm{f}^{\sigma} \mathrm{g}^{\Delta}, \\
\left(\frac{\mathrm{f}}{\mathrm{g}}\right)^{\Delta}=\frac{\mathrm{f}^{\Delta} \mathrm{g}-\mathrm{fg} \mathrm{g}^{\Delta}}{\mathrm{g} \mathrm{g}^{\sigma}}
\end{array}\right.
$$

Let $f: \mathbb{R} \rightarrow \mathbb{R}$ be continuously differentiable and suppose that $g: \mathbb{T} \rightarrow \mathbb{R}$ is delta differentiable. Then $\mathrm{f} \circ \mathrm{g}: \mathbb{T} \rightarrow \mathbb{R}$ is delta differentiable and the the next formula

$$
(f \circ g)^{\Delta}(t)=\left(\int_{0}^{1} f^{\prime}\left(g(t)+h \mu(t) g^{\Delta}(t)\right) d h\right) g^{\Delta}(t),
$$

holds. A special case of the chain rule is

$$
\left[\mathfrak{u}^{\lambda}(\mathrm{t})\right]^{\Delta}=\lambda \int_{0}^{1}\left[h u^{\sigma}(t)+(1-h) u(t)\right]^{\lambda-1} u^{\Delta}(t) d h .
$$

The integration by parts formula on time scale is given by

$$
\int_{a}^{b} u(t) v^{\Delta}(t) \Delta t=\left.u(t) v(t)\right|_{a} ^{b}-\int_{a}^{b} u^{\Delta}(t) v^{\sigma}(t) \Delta t
$$

Hölder inequality on time scale is given by

$$
\int_{a}^{b}|f(t) g(t)| \Delta t \leqslant\left(\int_{a}^{b}|f(t)|^{p} \Delta t\right)^{\frac{1}{p}}\left(\int_{a}^{b}|g(t)|^{q} \Delta t\right)^{\frac{1}{q}},
$$

where $p>1,1 / p+1 / q=1$ and $f, g \in C_{r d}\left([a, b]_{\mathbb{T}}, \mathbb{R}\right)$. The inequality (2.2) is reversed for $0<p<1$ or $p<0$. Minkowiski's inequality on time scale is given by

$$
\left(\int_{a}^{b}|h(x)||f(x)+g(x)|^{p} \Delta x\right)^{\frac{1}{p}} \leqslant\left(\int_{a}^{b}|h(x)||f(x)|^{p} \Delta x\right)^{\frac{1}{p}}+\left(\int_{a}^{b}|h(x)||g(x)|^{p} \Delta x\right)^{\frac{1}{p}},
$$

where $p>1$ and $f, g, h \in C_{r d}\left([a, b]_{\mathbb{T}}, \mathbb{R}\right)$. We need the following lemmas to prove our results.

Lemma 2.2 ([32]). Let $\mathbb{T}$ be a time scale with $a, x \in \mathbb{T}$ such that $x \geqslant a$. If $p \geqslant 1$, then

$$
\left(\int_{a}^{\sigma(x)} h(t) \Delta t\right)^{p} \leqslant p \int_{a}^{\sigma(x)} h(t)\left(\int_{a}^{\sigma(t)} h(z) \Delta z\right)^{p-1} \Delta t .
$$

Lemma 2.3 ([32] ). Let $\mathbb{T}$ be a time scale with $\mathrm{b}, x \in \mathbb{T}$ such that $\mathrm{b} \geqslant x$. If $\mathrm{p} \geqslant 1$, then

$$
\left(\int_{x}^{b} h(t) \Delta t\right)^{p} \leqslant p \int_{x}^{b} h(t)\left(\int_{t}^{b} h(z) \Delta z\right)^{p-1} \Delta t .
$$

Lemma 2.4 ([34] ). Let $\mathbb{T}$ be a time scale with $\mathrm{a}, \mathrm{b} \in \mathbb{T}$ and $\mathrm{h}, \mathrm{H}$ be nonnegative and $r$-continuous function defined on $[\mathrm{a}, \mathrm{b}]_{\mathbb{T}}$. Then

$$
\int_{a}^{b} H(t)\left(\int_{t}^{b} h(x) \Delta t\right) \Delta x=\int_{a}^{b} h(t)\left(\int_{a}^{\sigma(t)} H(x) \Delta x\right) \Delta t .
$$

The results obtained in this paper have numerous applications in the study of the oscillation of various classes of dynamic equations on time scales; see, for instance, [14, 16]. 


\section{Main results}

In this section, we will state and prove our main results. Throughout this paper, we will assume that the functions in the statements of the theorems are rd-continuous nonnegative functions defined on $\mathbb{I}=[a, \infty)_{\mathbb{T}}$ and the integrals considered are assumed to exist and finite and assume for the sake of conventions that $0 \cdot \infty=0$ and $0 / 0=0$. For any function $\phi: \mathbb{I} \rightarrow \mathbb{R}^{+}$, we will define $p^{\prime}$ by $1 / p+1 / p^{\prime}=1$, for $p>1$. Also the operator $\Phi: \mathbb{I} \rightarrow \mathbb{R}^{+}$by

$$
\Phi(x)=\int_{a}^{x} \phi(t) \Delta t
$$

Theorem 3.1. Let $\mathrm{p}>1$ and $\mathbb{T}$ be a time scale with $\mathrm{a} \in[0, \infty)_{\mathbb{T}}$. Let $\phi(\mathrm{x})$ be a positive and nondecreasing such that

$$
\sigma(x)\left|\phi^{\Delta}(x)\right| \leqslant A \phi(x), \text { and } \sigma(x) \phi^{\sigma}(x) \leqslant B \Phi^{\sigma}(x), \text { for all } x \in \mathbb{I} \text {. }
$$

Then

$$
\int_{a}^{\infty} \phi(x)\left(\frac{1}{\Phi^{\sigma}(x)} \int_{a}^{\sigma(x)} \phi(t) f(t) \Delta t\right)^{p} \Delta x \leqslant(A+B)^{p} \int_{a}^{\infty}\left(\frac{1}{\sigma(x)} \int_{a}^{\sigma(x)} \phi^{\frac{1}{p}}(t) f(t) \Delta t\right)^{p} \Delta x
$$

where $\mathrm{A}$ and $\mathrm{B}$ are positive constants and $\Phi(\infty)=\infty$.

Proof. For simplicity, we define $g(x)=\int_{a}^{x} \phi^{1 / p}(t) f(t) \Delta t$. This gives

$$
g^{\Delta}(x)=\phi^{\frac{1}{p}}(x) f(x),
$$

and then $f(x)=g^{\Delta}(x) \phi^{-1 / p}(x)$. The left hand side of (3.2) now can be written in terms of $g$ in the form

$$
\begin{aligned}
\int_{a}^{\infty} \phi(x)\left(\frac{1}{\Phi^{\sigma}(x)} \int_{a}^{\sigma(x)} \phi(t) f(t) \Delta t\right)^{p} \Delta x & =\int_{a}^{\infty} \phi(x)\left(\frac{1}{\Phi^{\sigma}(x)} \int_{a}^{\sigma(x)} \phi(t) g^{\Delta}(t) \phi^{-\frac{1}{p}}(t) \Delta t\right)^{p} \Delta x \\
& =\int_{a}^{\infty} \phi(x)\left(\frac{1}{\Phi^{\sigma}(x)} \int_{a}^{\sigma(x)} g^{\Delta}(t) \phi^{\frac{1}{p^{\prime}}}(t) \Delta t\right)^{p} \Delta x
\end{aligned}
$$

where $p^{\prime}$ is the conjugate of $p$ such that $1 / p+1 / p^{\prime}=1$; for $p>1$. Also integrating the term $\int_{a}^{\sigma(x)} g^{\Delta}(t) \phi^{1 / p^{\prime}}(t) \Delta t$ by parts with $u(t)=\phi^{1 / p^{\prime}}(t)$ and $v^{\Delta}(t)=g^{\Delta}(t)$, we obtain

$$
\begin{aligned}
\int_{a}^{\sigma(x)} g^{\Delta}(t) \phi^{\frac{1}{p^{\prime}}}(t) \Delta t & =\left.g(t) \phi^{\frac{1}{p^{\prime}}}(t)\right|_{a} ^{\sigma(x)}-\int_{a}^{\sigma(x)} g^{\sigma}(t)\left(\phi^{\frac{1}{p^{\prime}}}(t)\right)^{\Delta} \Delta t \\
& =g^{\sigma}(x)\left(\phi^{\sigma}(x)\right)^{\frac{1}{p^{\prime}}}-\int_{a}^{\sigma(x)} g^{\sigma}(t)\left(\phi^{\frac{1}{p^{\prime}}}(t)\right)^{\Delta} \Delta t .
\end{aligned}
$$

By (3.3) and (3.4), we have

$$
\begin{aligned}
& \int_{a}^{\infty} \phi(x)\left(\frac{1}{\Phi^{\sigma}(x)} \int_{a}^{\sigma(x)} \phi(t) f(t) \Delta t\right)^{p} \Delta x \\
& \quad=\int_{a}^{\infty} \phi(x)\left(\frac{1}{\Phi^{\sigma}(x)}\left[g^{\sigma}(x)\left(\phi^{\sigma}(x)\right)^{\frac{1}{p^{\prime}}}-\int_{a}^{\sigma(x)} g^{\sigma}(t)\left(\phi^{\frac{1}{p^{\prime}}}(\mathrm{t})\right)^{\Delta} \Delta t\right]\right)^{p} \Delta x
\end{aligned}
$$


Applying Minkowski's inequality (2.3), we see

$$
\begin{aligned}
& \left(\int_{a}^{\infty} \phi(x)\left[\frac{1}{\Phi^{\sigma}(x)} \int_{a}^{\sigma(x)} \phi(t) f(t) \Delta t\right]^{p} \Delta x\right)^{\frac{1}{p}} \\
& \leqslant\left(\int_{a}^{\infty} \phi(x)\left[\frac{g^{\sigma}(x)\left(\phi^{\sigma}(x)\right)^{\frac{1}{p}}}{\Phi^{\sigma}(x)}\right]^{p} \Delta x\right)^{\frac{1}{p}}+\left(\int_{a}^{\infty} \phi(x)\left[\frac{1}{\Phi^{\sigma}(x)} \int_{a}^{\sigma(x)} g^{\sigma}(t)\left|\left(\phi^{\frac{1}{p^{\prime}}}(t)\right)^{\Delta}\right| \Delta t\right]^{p} \Delta x\right)^{\frac{1}{p}} .
\end{aligned}
$$

Applying the chain rule (2.1) (noting that $\phi(x)$ is nondecreasing function), we see

$$
\begin{aligned}
\left(\phi^{\frac{1}{p^{\prime}}}(t)\right)^{\Delta} & =\frac{1}{p^{\prime}}\left(\int_{0}^{1}\left[h \phi^{\sigma}(t)+(1-h) \phi(t)\right]^{\frac{1}{p^{\prime}}-1} d h\right) \phi^{\Delta}(t) \\
& =\frac{1}{p^{\prime}} \int_{0}^{1} \frac{d h}{\left(h \phi^{\sigma}(t)+(1-h) \phi(t)\right)^{1-\frac{1}{p^{\prime}}}} \phi^{\Delta}(t) \\
& \leqslant \frac{1}{p^{\prime}} \int_{0}^{1} \frac{d h}{(h \phi(t)+(1-h) \phi(t))^{1-\frac{1}{p^{\prime}}}} \phi^{\Delta}(t) \\
& =\frac{1}{p^{\prime}} \phi^{\frac{1}{p^{\prime}}-1}(t) \phi^{\Delta}(t)=\frac{1}{p^{\prime}} \phi^{-\frac{1}{p}}(t) \phi^{\Delta}(t) .
\end{aligned}
$$

Now by using (3.5), we have

$$
\begin{aligned}
& \left(\int_{a}^{\infty} \phi(x)\left[\frac{1}{\Phi^{\sigma}(x)} \int_{a}^{\sigma(x)} \phi(t) f(t) \Delta t\right]^{p} \Delta x\right)^{\frac{1}{p}} \\
& \leqslant\left(\int_{a}^{\infty} \phi(x)\left[\frac{g^{\sigma}(x)\left(\phi^{\sigma}(x)\right)^{\frac{1}{p^{\prime}}}}{\Phi^{\sigma}(x)}\right]^{p} \Delta x\right)^{\frac{1}{p}} \\
& \quad+\frac{1}{p^{\prime}}\left(\int_{a}^{\infty} \phi(x)\left[\frac{1}{\Phi^{\sigma}(x)} \int_{a}^{\sigma(x)} g^{\sigma}(t) \phi^{-\frac{1}{p}}(t)\left|\phi^{\Delta}(t)\right| \Delta t\right]^{p} \Delta x\right)^{\frac{1}{p}} .
\end{aligned}
$$

Since $\phi(x)$ is nondecreasing we have by using (3.1), that

$$
\begin{aligned}
\int_{a}^{\infty} \phi(x)\left(\frac{g^{\sigma}(x)\left(\phi^{\sigma}(x)\right)^{\frac{1}{p^{\prime}}}}{\Phi^{\sigma}(x)}\right)^{p} \Delta x & \leqslant \int_{a}^{\infty} \phi^{\sigma}(x)\left(\frac{B g^{\sigma}(x)\left(\phi^{\sigma}(x)\right)^{\frac{1}{p^{\prime}}}}{\sigma(x) \phi^{\sigma}(x)}\right)^{p} \Delta x \\
& =\int_{a}^{\infty} \phi^{\sigma}(x)\left(\frac{B g^{\sigma}(x)\left(\phi^{\sigma}(x)\right)^{-\frac{1}{p}}}{\sigma(x)}\right)^{p} \Delta x=B^{p} \int_{a}^{\infty}\left(\frac{g^{\sigma}(x)}{\sigma(x)}\right)^{p} \Delta x .
\end{aligned}
$$

Let

$$
\omega(t)=g^{\sigma}(t) \phi(t)^{-\frac{1}{p}}\left|\phi^{\Delta}(t)\right| \text { and } \Omega(x)=\int_{a}^{x} \omega(t) \Delta t .
$$

From the definition (3.9), Lemma 2.2, and Lemma 2.4, we get

$$
\begin{aligned}
& \int_{a}^{\infty} \phi(x)\left(\frac{1}{\Phi^{\sigma}(x)} \int_{a}^{\sigma(x)} g^{\sigma}(t) \phi^{-\frac{1}{p}}(t)\left|\phi^{\Delta}(t)\right| \Delta t\right)^{p} \Delta x \\
& \leqslant \int_{a}^{\infty} \phi(x)\left(\frac{1}{\Phi^{\sigma}(x)} \int_{a}^{\sigma(x)} g^{\sigma}(t) \phi^{-\frac{1}{p}}(t)\left|\phi^{\Delta}(t)\right| \Delta t\right)^{p} \Delta x
\end{aligned}
$$




$$
\begin{aligned}
& =\int_{a}^{\infty} \phi(x)\left(\frac{1}{\Phi^{\sigma}(x)} \int_{a}^{\sigma(x)} \omega(t) \Delta t\right)^{p} \Delta x \\
& \leqslant p \int_{a}^{\infty} \phi(x)\left(\Phi^{\sigma}(x)\right)^{-p}\left(\int_{a}^{\sigma(x)} \omega(t)\left(\int_{a}^{\sigma(t)} \omega(z) \Delta z\right)^{p-1} \Delta t\right) \Delta x \\
& =p \int_{a}^{\infty} \phi(x)\left(\Phi^{\sigma}(x)\right)^{-p} \int_{a}^{\sigma(x)} \omega(t)(\Omega(\sigma(t)))^{p-1} \Delta t \Delta x \\
& \leqslant p \int_{a}^{\infty} \omega(t)(\Omega(\sigma(t)))^{p-1} \int_{t}^{\infty} \frac{\phi(x)}{\left(\Phi^{\sigma}(x)\right)^{p} \Delta x \Delta t .}
\end{aligned}
$$

Since $\Phi(x) \leqslant \Phi^{\sigma}(x)$, then

$$
\frac{1}{\left(h \Phi^{\sigma}(x)+(1-h) \Phi(x)\right)^{p}} \geqslant \frac{1}{\left(h \Phi^{\sigma}(x)+(1-h) \Phi^{\sigma}(x)\right)^{p}}
$$

and since $p>1$,

$$
\frac{(1-p)}{\left(h \Phi^{\sigma}(x)+(1-h) \Phi(x)\right)^{p}} \leqslant \frac{(1-p)}{\left(h \Phi^{\sigma}(x)+(1-h) \Phi^{\sigma}(x)\right)^{p}} .
$$

Applying the chain rule (2.1), we get

$$
\begin{aligned}
\left(\Phi(x)^{1-p}\right)^{\Delta} & =(1-p) \int_{0}^{1}\left(h \Phi^{\sigma}(x)+(1-h) \Phi(x)\right)^{-p} \operatorname{dh}(\Phi(x))^{\Delta} \\
& \leqslant(1-p) \int_{0}^{1} \frac{d h(\Phi(x))^{\Delta}}{\left(h \Phi^{\sigma}(x)+(1-h) \Phi^{\sigma}(x)\right)^{p}}=(1-p) \phi(x)\left(\Phi^{\sigma}(x)\right)^{-p} .
\end{aligned}
$$

Integrating the last inequality from $t$ to $\infty$, we obtain

$$
\int_{t}^{\infty} \phi(x)\left(\Phi^{\sigma}(x)\right)^{-p} \Delta x \leqslant \frac{1}{(p-1)}(\Phi(t))^{1-p} .
$$

Substituting into (3.10), we obtain

$$
\begin{aligned}
& \int_{a}^{\infty} \phi(x)\left\{\frac{1}{\Phi^{\sigma}(x)} \int_{a}^{\sigma(x)} g^{\sigma}(t) \phi(t)^{-\frac{1}{p}}\left|\phi^{\Delta}(t)\right| \Delta t\right\}^{p} \Delta x \\
& \leqslant \frac{p}{(p-1)} \int_{a}^{\infty} \omega(t)(\Omega(\sigma(t)))^{p-1} \Phi(t)^{1-p} \Delta t=\frac{p}{(p-1)} \int_{a}^{\infty} \omega(t)\left(\frac{\Omega(\sigma(t))}{\Phi(t)}\right)^{p-1} \Delta t .
\end{aligned}
$$

Thus by (3.9), we have

$$
\int_{a}^{\infty} \phi(x)\left(\frac{\Omega(\sigma(x))}{\Phi(x)}\right)^{p} \Delta x \leqslant \frac{p}{p-1} \int_{a}^{\infty} \omega(t)\left(\frac{\Omega(\sigma(t))}{\Phi(t)}\right)^{p-1} \Delta t
$$

Next, writing $\omega(t)=\left(\omega(t) \phi^{-1 / p^{\prime}}(t)\right) \phi^{1 / p^{\prime}}(t)$ and applying Hölder's inequality (2.2) with exponents $p$ and $p^{\prime}$, we have

$$
\int_{a}^{\infty} \omega(t)\left(\frac{\Omega(\sigma(t))}{\Phi(t)}\right)^{p-1} \Delta t \leqslant\left(\int_{a}^{\infty}\left[\omega(t) \phi^{-\frac{1}{p^{\prime}}(t)}\right]^{p} \Delta t\right)^{\frac{1}{p}}\left(\int_{a}^{\infty} \phi(t)\left[\frac{\Omega(\sigma(t))}{\Phi(t)}\right]^{p} \Delta t\right)^{\frac{1}{p^{\prime}}} .
$$

We can obtain from (3.11) and (3.12) that

$$
\int_{a}^{\infty} \phi(x)\left(\frac{\Omega(\sigma(x))}{\Phi(x)}\right)^{p} \Delta x \leqslant \frac{p}{p-1}\left(\int_{a}^{\infty}\left[\omega(t) \phi^{-\frac{1}{p^{\prime}}(t)}\right]^{p} \Delta t\right)^{\frac{1}{p}}\left(\int_{a}^{\infty} \phi(t)\left[\frac{\Omega(\sigma(t))}{\Phi(t)}\right]^{p} \Delta t\right)^{\frac{1}{p^{\prime}}} .
$$


By combining (3.9) and (3.1), we see

$$
\omega(t) \phi^{-\frac{1}{p^{\prime}}}(t)=g^{\sigma}(t) \phi^{-\frac{1}{p}}(t)\left|\phi^{\Delta}(t)\right| \phi^{-\frac{1}{p^{\prime}}}(t)=g^{\sigma}(t)\left|\phi^{\Delta}(t)\right| \phi^{-1}(t) \leqslant A \frac{g^{\sigma}(t)}{\sigma(t)} .
$$

Substituting into (3.13) and dividing by the last term, we get

$$
\left(\int_{a}^{\infty} \phi(x)\left[\frac{\Omega(\sigma(x))}{\Phi(x)}\right]^{p} \Delta x\right)^{\frac{1}{p}} \leqslant \frac{p}{p-1}\left(\int_{a}^{\infty}\left[A \frac{g^{\sigma}(t)}{\sigma(t)}\right]^{p} \Delta t\right)^{\frac{1}{p}} .
$$

So

$$
\int_{a}^{\infty} \phi(x)\left[\frac{\Omega(\sigma(x))}{\Phi(x)}\right]^{p} \Delta x \leqslant A^{p}\left(\frac{p}{p-1}\right)^{p} \int_{a}^{\infty}\left[\frac{g^{\sigma}(t)}{\sigma(t)}\right]^{p} \Delta t .
$$

Substituting (3.14) and (3.8) into (3.7), we get

$$
\begin{aligned}
& \left(\int_{a}^{\infty} \phi(x)\left[\frac{1}{\Phi^{\sigma}(x)} \int_{a}^{\sigma(x)} \phi(t) f(t) \Delta t\right]^{p} \Delta x\right)^{\frac{1}{p}} \\
& \leqslant\left(B^{p} \int_{a}^{\infty}\left[\frac{g^{\sigma}(x)}{\sigma(x)}\right]^{p} \Delta x\right)^{\frac{1}{p}}+\frac{1}{p^{\prime}}\left(A^{p}\left(\frac{p}{p-1}\right)^{p} \int_{a}^{\infty}\left[\frac{g^{\sigma}(x)}{\sigma(x)}\right]^{p} \Delta x\right)^{\frac{1}{p}}=(B+A)\left(\int_{a}^{\infty}\left[\frac{g^{\sigma}(x)}{\sigma(x)}\right]^{p} \Delta x\right)^{\frac{1}{p}},
\end{aligned}
$$

which is the desired inequality (3.2). The proof is complete.

Remark 3.2. As a special case of Theorem 3.1 when $\mathbb{T}=\mathbb{R}$, we have the inequality (1.7).

In the following, we consider the case when $\mathbb{T}=\mathbb{N}$, and formulate the discrete version of (1.7).

If $p>1,(n+1)|\Delta \phi(n)| \leqslant A \phi(n)$, and $(n+1) \phi(n+1) \leqslant B \Phi(n+1)$, then

$$
\sum_{n=1}^{\infty} \phi(n)\left(\frac{1}{\Phi(n+1)} \sum_{k=1}^{n} \phi(k) f(k)\right)^{p} \leqslant(A+B)^{p} \sum_{n=1}^{\infty}\left(\frac{1}{n+1} \sum_{k=1}^{n} \phi^{\frac{1}{p}}(k) f(k)\right)^{p} .
$$

To prove the following theorem, we will assume that there exists a constant $m \geqslant 1$ such that $\Phi^{\sigma}(t) \leqslant$ $m \Phi(t)$.

Theorem 3.3. Let $\mathbb{T}$ be a time scale with $\mathrm{a} \in[0, \infty)_{\mathbb{T}}$ and $\mathrm{p}>1$. Let $\phi(\mathrm{x})$ be a positive and nondecreasing function. Then

$$
\int_{a}^{\infty} \phi(x)\left(\int_{\sigma(x)}^{\infty} \frac{\phi(t) f(t)}{\Phi(t)} \Delta t\right)^{p} \Delta x \leqslant(B+C p m)^{p} \int_{a}^{\infty}\left(\int_{x}^{\infty} \frac{\phi^{\frac{1}{p}}(t) f(t)}{t} \Delta t\right)^{p} \Delta x,
$$

where $\mathrm{C}=1+\mathrm{A}+\mathrm{B}$.

Proof. Set $\Lambda(x)=\int_{x}^{\infty} \phi^{1 / p}(t) f(t) / t \Delta t$. Hence $\Lambda^{\Delta}(x)=-\phi^{1 / p}(x) f(x) / x<0$, and consequently $f(x)=$ $-x \phi^{-1 / p}(x) \wedge^{\Delta}(x)$. This implies that

$$
\int_{\sigma(x)}^{\infty} \frac{\phi(t) f(t)}{\Phi(t)} \Delta t=-\int_{\sigma(x)}^{\infty} \frac{t \phi^{\frac{1}{p^{\prime}}}(t)}{\Phi(t)} \Lambda^{\Delta}(t) \Delta t
$$

Integrating by parts with $u(t)=t \phi^{1 / p^{\prime}}(t) / \Phi(t)$ and $v^{\Delta}(t)=\Lambda^{\Delta}(t)$, we get

$$
\int_{\sigma(x)}^{\infty} \frac{\phi(t) f(t)}{\Phi(t)} \Delta t=\frac{\sigma(x)(\phi(\sigma(x)))^{\frac{1}{p^{\prime}}}}{\Phi^{\sigma}(x)} \Lambda^{\sigma}(x)+\int_{\sigma(x)}^{\infty}\left(\frac{t \phi^{\frac{1}{p^{\prime}}}(t)}{\Phi(t)}\right)^{\Delta} \Lambda^{\sigma}(t) \Delta t
$$




$$
\leqslant \frac{\sigma(x)(\phi(\sigma(x)))^{\frac{1}{p^{\prime}}}}{\Phi^{\sigma}(x)} \Lambda^{\sigma}(x)+\int_{\sigma(x)}^{\infty}\left|\left(\frac{t \phi^{\frac{1}{p^{\prime}}}(\mathrm{t})}{\Phi(\mathrm{t})}\right)^{\Delta}\right| \Lambda^{\sigma}(\mathrm{t}) \Delta \mathrm{t}
$$

This implies that

$$
\begin{aligned}
& \int_{a}^{\infty} \phi(x)\left(\int_{\sigma(x)}^{\infty} \frac{\phi(t) f(t)}{\Phi(t)} \Delta t\right)^{p} \Delta x \\
& \quad \leqslant \int_{a}^{\infty} \phi(x)\left(\frac{\sigma(x)(\phi(\sigma(x)))^{\frac{1}{p^{\prime}}}}{\Phi^{\sigma}(x)} \Lambda^{\sigma}(x)+\int_{\sigma(x)}^{\infty}\left|\left(\frac{t \phi^{\frac{1}{p^{\prime}}(t)}}{\Phi(t)}\right)^{\Delta}\right| \Lambda^{\sigma}(t) \Delta t\right)^{p} \Delta x .
\end{aligned}
$$

Applying Minkowski's inequality (2.3), we see

$$
\begin{aligned}
\left(\int_{a}^{\infty} \phi(x)\left[\int_{\sigma(x)}^{\infty} \frac{\phi(t) f(t)}{\Phi(t)} \Delta t\right]^{p} \Delta x\right)^{\frac{1}{p}} & \leqslant\left(\int_{a}^{\infty} \phi(x)\left[\frac{\sigma(x)(\phi(\sigma(x)))^{\frac{1}{p^{\prime}}}}{\Phi^{\sigma}(x)} \Lambda^{\sigma}(x)\right]^{p} \Delta x\right)^{\frac{1}{p}} \\
& +\left(\int_{a}^{\infty} \phi(x)\left(\int_{\sigma(x)}^{\infty}\left|\left(\frac{t \phi^{\frac{1}{p^{\prime}}}(t)}{\Phi(t)}\right)^{\Delta}\right| \Lambda^{\sigma}(t) \Delta t\right)^{p} \Delta x\right)^{\frac{1}{p}} .
\end{aligned}
$$

Since $\phi(x)$ is nondecreasing, $\Lambda(x)$ is nonincreasing so $\Lambda^{\sigma}(x) \leqslant \Lambda(x)$ and by (3.1), we get

$$
\begin{aligned}
\int_{a}^{\infty} \phi(x)\left(\frac{\sigma(x)(\phi(\sigma(x)))^{\frac{1}{p^{\prime}}}}{\Phi^{\sigma}(x)} \Lambda^{\sigma}(x)\right)^{p} \Delta x & \leqslant \int_{a}^{\infty} \phi^{\sigma}(x)\left(\frac{B \sigma(x)(\phi(\sigma(x)))^{\frac{1}{p^{\prime}}}}{\sigma(x) \phi^{\sigma}(x)} \Lambda^{\sigma}(x)\right)^{p} \Delta x \\
& =\int_{a}^{\infty} \phi^{\sigma}(x)\left[B(\phi(\sigma(x)))^{-\frac{1}{p}} \Lambda^{\sigma}(x)\right]^{p} \Delta x \\
& =B^{p} \int_{a}^{\infty}\left[\Lambda^{\sigma}(x)\right]^{p} \Delta x \leqslant B^{p} \int_{a}^{\infty}[\Lambda(x)]^{p} \Delta x .
\end{aligned}
$$

By using the quotient and product rules (2.1) and (3.6), we have

$$
\begin{aligned}
\left(\frac{\mathrm{t} \phi^{\frac{1}{p^{\prime}}}(\mathrm{t})}{\Phi(\mathrm{t})}\right)^{\Delta} & =\frac{\Phi(\mathrm{t})\left(\mathrm{t} \phi^{\frac{1}{p^{\prime}}}(\mathrm{t})\right)^{\Delta}-\mathrm{t} \phi^{\frac{1}{p^{\prime}}}(\mathrm{t}) \Phi^{\Delta}(\mathrm{t})}{\Phi(\mathrm{t}) \Phi^{\sigma}(\mathrm{t})} \\
& =\frac{\Phi(\mathrm{t}) \phi^{\frac{1}{p^{\prime}}}(\mathrm{t})+\sigma(\mathrm{t}) \Phi(\mathrm{t})\left(\phi^{\frac{1}{p^{\prime}}}(\mathrm{t})\right)^{\Delta}-\mathrm{t} \phi^{\frac{1}{p^{\prime}}+1}(\mathrm{t})}{\Phi(\mathrm{t}) \Phi^{\sigma}(\mathrm{t})} \\
& \leqslant \frac{\phi^{\frac{1}{p^{\prime}}}(\mathrm{t})}{\Phi^{\sigma}(\mathrm{t})}+\frac{\sigma(\mathrm{t}) \phi^{\Delta}(\mathrm{t}) \phi^{\frac{1}{p^{\prime}}-1}(\mathrm{t})}{\mathrm{p}^{\prime} \Phi^{\sigma}(\mathrm{t})}-\frac{\mathrm{t} \phi^{\frac{1}{p^{\prime}}+1}(\mathrm{t})}{\Phi(\mathrm{t}) \Phi^{\sigma}(\mathrm{t})} \\
& =\frac{\mathrm{t} \phi^{\frac{1}{p^{\prime}}}(\mathrm{t})}{\Phi^{\sigma}(\mathrm{t})}\left[\frac{1}{\mathrm{t}}+\frac{\sigma(\mathrm{t}) \phi^{\Delta}(\mathrm{t})}{\mathrm{t} \phi(\mathrm{t})}-\frac{\phi(\mathrm{t})}{\Phi(\mathrm{t})}\right]
\end{aligned}
$$

Thus, we have

$$
\left|\left(\frac{\mathrm{t} \phi^{\frac{1}{\mathrm{p}^{\prime}}}(\mathrm{t})}{\Phi(\mathrm{t})}\right)^{\Delta}\right| \leqslant \frac{\mathrm{t} \phi^{\frac{1}{\mathrm{p}^{\prime}}}(\mathrm{t})}{\Phi^{\sigma}(\mathrm{t})}\left[\frac{1}{\mathrm{t}}+\frac{\sigma(\mathrm{t})\left|\phi^{\Delta}(\mathrm{t})\right|}{\mathrm{p}^{\prime} \mathrm{t} \phi(\mathrm{t})}+\frac{\phi(\mathrm{t})}{\Phi(\mathrm{t})}\right] \leqslant \frac{\mathrm{t} \phi^{\frac{1}{\mathrm{p}^{\prime}}}(\mathrm{t})}{\Phi^{\sigma}(\mathrm{t})}\left[\frac{1}{\mathrm{t}}+\frac{\sigma(\mathrm{t})\left|\phi^{\Delta}(\mathrm{t})\right|}{\mathrm{p}^{\prime} \mathrm{t} \phi(\mathrm{t})}+\frac{\phi^{\sigma}(\mathrm{t})}{\Phi(\mathrm{t})}\right] .
$$


Since $\phi(t)$ is nondecreasing and by the assumption (3.1), we have

$$
\left|\left(\frac{t \phi^{\frac{1}{p^{\prime}}}(t)}{\Phi(t)}\right)^{\Delta}\right| \leqslant \frac{t \phi^{\frac{1}{p^{\prime}}}(t)}{\Phi^{\sigma}(t)}\left(\frac{1}{t}+\frac{A}{p^{\prime} t}+\frac{B}{t}\right)<C \frac{\phi^{\frac{1}{p^{\prime}}}(t)}{\Phi^{\sigma}(t)} \leqslant C \frac{\phi^{\frac{1}{p^{\prime}}}(t)}{\Phi(t)}
$$

where $C=1+A+B$. Since $\Lambda(t)$ is nonincreasing, we get

$$
\int_{a}^{\infty} \phi(x)\left(\int_{\sigma(x)}^{\infty}\left|\left(\frac{t \phi^{\frac{1}{p^{\prime}}}(t)}{\Phi(t)}\right)^{\Delta}\right| \Lambda^{\sigma}(t) \Delta t\right)^{p} \Delta x \leqslant C \int_{a}^{\infty} \phi(x)\left(\int_{\sigma(x)}^{\infty} \frac{\phi^{\frac{1}{p^{\prime}}}(t)}{\Phi(t)} \Lambda(t) \Delta t\right)^{p} \Delta x .
$$

Let

$$
\psi(t)=\frac{\phi^{\frac{1}{p^{\prime}}}(t)}{\Phi(t)} \Lambda(t) \text { and } \Psi(t)=\int_{t}^{\infty} \psi(z) \Delta z
$$

Then

$$
\Psi^{\Delta}(\mathrm{t})=-\psi(\mathrm{t})=-\frac{\phi^{\frac{1}{p^{\prime}}}(\mathrm{t})}{\Phi(\mathrm{t})} \Lambda(\mathrm{t})<0
$$

Now, from (3.19) and since $\Psi(t)$ is nonincreasing we have $\Psi(\sigma(t)) \leqslant \Psi(t)$, we get

$$
\int_{a}^{\infty} \phi(x)\left(\int_{\sigma(x)}^{\infty} \frac{\phi^{\frac{1}{p^{\prime}}}(\mathrm{t})}{\Phi(\mathrm{t})} \Lambda(\mathrm{t}) \Delta \mathrm{t}\right)^{\mathrm{p}} \Delta \mathrm{x}=\int_{\mathrm{a}}^{\infty} \phi(\mathrm{x})(\Psi(\sigma(\mathrm{x})))^{\mathrm{p}} \Delta \mathrm{x} \leqslant \int_{\mathrm{a}}^{\infty} \phi(\mathrm{x})(\Psi(\mathrm{x}))^{\mathrm{p}} \Delta \mathrm{x} .
$$

Applying Lemma (2.3), we get

$$
\int_{a}^{\infty} \phi(x)(\Psi(x))^{p} \Delta x \leqslant p \int_{a}^{\infty} \phi(x)\left(\int_{x}^{\infty} \psi(t)(\Psi(x))^{p-1} \Delta t\right) \Delta x .
$$

Integrating by parts with $u^{\Delta}(x)=\phi(x), v(x)=\int_{x}^{\infty} \psi(t)\left(\Psi(t)^{p-1} \Delta t\right.$ and by using the assumption $\Phi^{\sigma}(x) \leqslant$ $m \Phi(x)$, we get

$$
\begin{aligned}
\int_{a}^{\infty} \phi(x)\left(\int_{x}^{\infty} \psi(t) \Psi(t)^{p-1} \Delta t\right) \Delta x & =\left.\Phi(x) \int_{x}^{\infty} \psi(t) \Psi(t)^{p-1}\right|_{a} ^{\infty}+\int_{a}^{\infty} \Phi^{\sigma}(x)\left(-\int_{x}^{\infty} \psi(t) \Psi(t)^{p-1} \Delta t\right)^{\Delta} \Delta x \\
& =\int_{a}^{\infty} \Phi^{\sigma}(x) \psi(x)(\Psi(x))^{p-1} \Delta x \leqslant m \int_{a}^{\infty} \Phi(x) \psi(x)(\Psi(x))^{p-1} \Delta x .
\end{aligned}
$$

Thus

$$
\int_{a}^{\infty} \phi(x)(\Psi(x))^{p} \Delta x \leqslant p m \int_{a}^{\infty} \Phi(x) \psi(x)(\Psi(x))^{p-1} \Delta x=p m \int_{a}^{\infty} \Phi(x) \psi(x) \phi^{-\frac{1}{p^{\prime}}}(x) \phi^{\frac{1}{p^{\prime}}}(x) \Psi^{p-1}(x) \Delta x .
$$

Applying Hölder inequality (2.2) with exponents $p$ and $p^{\prime}$, we obtain

$$
\int_{a}^{\infty} \phi(x)(\Psi(x))^{p} \Delta x \leqslant p m\left(\int_{a}^{\infty}\left[\Phi(x) \psi(x) \phi^{-\frac{1}{p^{\prime}}(x)}\right]^{p} \Delta x\right)^{\frac{1}{p}}\left(\int_{a}^{\infty} \phi(x)(\Psi(x))^{p} \Delta x\right)^{\frac{1}{p^{\prime}}} .
$$

From the definition (3.19) and dividing by the last term, we have

$$
\int_{a}^{\infty} \phi(x)(\Psi(t))^{p} \Delta x \leqslant(p m)^{p} \int_{a}^{\infty} \Lambda(x)^{p} \Delta x
$$


Substituting (3.18) and (3.19) into (3.20), we see

$$
\int_{a}^{\infty} \phi(x)\left(\int_{\sigma(x)}^{\infty}\left|\left(\frac{t \phi^{\frac{1}{p^{\prime}}}(\mathrm{t})}{\Phi(\mathrm{t})}\right)^{\Delta}\right| \Lambda^{\sigma}(\mathrm{t}) \Delta \mathrm{t}\right)^{\mathrm{p}} \Delta \mathrm{x} \leqslant(\mathrm{Cpm})^{\mathrm{p}} \int_{a}^{\infty} \Lambda(\mathrm{x})^{\mathrm{p}} \Delta x .
$$

Substituting (3.17) and (3.21) into (3.16), we see

$$
\begin{aligned}
\left(\int_{a}^{\infty} \phi(x)\left[\int_{\sigma(x)}^{\infty} \frac{\phi(t) f(t)}{\Phi(t)} \Delta t\right]^{p} \Delta x\right)^{\frac{1}{p}} & \leqslant\left(B^{p} \int_{a}^{\infty} \Lambda(x)^{p} \Delta x\right)^{\frac{1}{p}}+\left((C p m)^{p} \int_{a}^{\infty} \Lambda(x)^{p} \Delta x\right)^{\frac{1}{p}} \\
& =(B+C p m)\left(\int_{a}^{\infty} \Lambda(x)^{p} \Delta x\right)^{\frac{1}{p}}
\end{aligned}
$$

which is the desired inequality (3.15). The proof is complete.

Remark 3.4. As a special case of Theorem 3.3 when $\mathbb{T}=\mathbb{R}$ and $m=1$, we have the inequality (1.8).

In the following we consider the case when $\mathbb{T}=\mathbb{N}$, and formulate the discrete version of (1.8).

Corollary 3.5. If $p>1,(n+1)|\Delta \phi(n)| \leqslant A \phi(n),(n+1) \phi(n+1) \leqslant B \Phi(n)$, then

$$
\sum_{n=1}^{\infty} \phi(n)\left(\sum_{k=n+1}^{\infty} \frac{\phi(k) f(k)}{\Phi(k)}\right)^{p} \leqslant(C p m+B)^{p} \sum_{n=1}^{\infty}\left(\sum_{k=n}^{\infty} \frac{\phi(k)^{1 / p} f(k)}{k}\right)^{p}
$$

where $\mathrm{C}=1+\mathrm{A}+\mathrm{B}$.

\section{Conclusion}

In the context of this article, we presented generalizations of weighted dynamic inequalities of Hardy's type on time scales. From these inequalities, as particular cases, we formulated some integral and new discrete inequalities. The technique is based on the applications of well-known inequalities and tools from time scale calculus. For future work, we can present such inequalities by using Rieman-Liouville type fractional integrals and fractional derivatives on time scales. It will also be very interesting to present such inequalities on quantum calculus.

\section{References}

[1] H. A. Abd El-Hamid, H. M. Rezk, A. M. Ahmed, G. AlNemer, M. Zakarya, H. A. El Saify, Dynamic Inequalities in Quotients with General Kernels and Measures, J. Funct. Spaces, 2020 (2020), 12 pages. 1

[2] R. P. Agarwal, D. O’Regan, S. H. Saker, Dynamic Inequalities on Time Scales, Springer, Switzerland, (2014). 1

[3] R. P. Agarwal, D. O’Regan, S. H. Saker, Hardy Type Inequalities on Time Scales, Springer, Switzerland, (2016). 1

[4] R. P. Agarwal, A.-A. Hyder, M. Zakarya, G. AlNemer, C. Cesarano, D. Assante, Exact solutions for a class of Wicktype stochastic (3+1)-dimensional modified Benjamin-Bona-Mahony equations, Axioms, 8 (2019), 15 pages.

[5] R. P. Agarwal, A.-A. Hyder, M. Zakarya, Well-posedness of stochastic modified Kawahara equation, Adv. Differ. Equ., 2020 (2020), 10 pages.

[6] A. M. Ahmed, G. AlNemer, M. Zakarya, H. M. Rezk, Some Dynamic Inequalities of Hilbert's Type, J. Funct. Spaces, 2020 (2020), 13 pages. 1

[7] G. AlNemer, M. Zakarya, H. A. Abd El-Hamid, M. R. Kenawy, H. M. Rezk, Dynamic Hardy-type inequalities with non-conjugate parameters, Alex. Eng. J., (2020), 1-10 1

[8] G. Bennett, Some elementary inequalities, Quart. J. Math. Oxford Ser., 38 (1987), 401-425. 1

[9] G. Bennett, Some elementary inequalities II, Quart. J. Math. Oxford Ser., 39 (1988), 385-400.

[10] G. Bennett, K.-G. Grosse-Erdmann, Weighted Hardy inequalities for decreasing sequences and functions, Math. Ann., 334 (2006), 489-531. 1, 1

[11] R. Bibi, M. Bohner, J. Pečarić, S. Varošanec, Minkowski and Beckenbach-Dresher inequalities and functionals on time scales, J. Math. Inequal., 7 (2013), 299-312. 1 
[12] M. Bohner, A. Peterson, Dynamic Equations on Time Scales: An Introduction with Applications, Birkhäuser, Boston, (2001). 2

[13] M. Bohner, A. Peterson, Advanced in Dynamic Equations on Time Scales, Birkhäuser, Boston, (2003). 1, 2

[14] M. Bohner, T. Li, Kamenev type criteria for nonlinear damped dynamic equations, Sci. China Math., 58 (2015), $1445-1452$. 2

[15] M. Bohner, S. G. Georgiev, Multiple integration on time scales: multivariable dynamic calculus on time scales, Springer, Switzerland, (2016), 449-515. 2

[16] M. Bohner, T. S. Hassan, T. Li, Fite-Hille-Wintner-type oscillation criteria for second-order half-linear dynamic equations with deviating arguments, Indag. Math., 29 (2018), 548-560. 2

[17] F. Carravetta, A. Germani, M. K. Shuakayev, A new suboptimal approach to the filtering problem for bilinear stochastic differential systems, SIAM J. Control Optim., 38 (2000), 1171-1203 1

[18] E. T. Copson, Note on series of positive terms, J. London. Math. Soc., 2 (1927), 9-12. 1

[19] E. T. Copson, Note on series of positive terms, J. London. Math. Soc., 3 (1928), 49-51. 1

[20] E. T. Copson, Some integral inequalities, Proc. Roy. Soc. Edinburgh Sect. A, 75 (1976), 157-164. 1

[21] H. A. Ghany, A.-A. Hyder, M. Zakarya, Exact solutions of stochastic fractional Korteweg de-Vries equation with conformable derivatives, Chin. Phys. B, 29 (2020).

[22] G. H. Hardy, Note on a theorem of Hilbert, Math. Z., 6 (1920), 314-317. 1

[23] G. H. Hardy, Notes on some points in the integral calculus (LX): An inequality between integrals, Messenger Math., 54 (1925), 150-156. 1, 1

[24] G. H. Hardy, J. E. Littlewood, G. Pólya, Inequalities second edition, Cambridge University Press, Cambridge, (1934). 1

[25] A. Kufner, L.-E. Persson, Weighted Inequalities of Hardy Type, World scientific Publishing Co., (2003). 1

[26] L. Leindler, Generalization of inequalities of Hardy and Littlewood, Acta Sci. Math., 31 (1970), 279-285. 1

[27] R. N. Mohapatra, K. Vajravelu, Integral inequalities resembling Copson's inequality, J. Austral. Math. Soc., 48 (1990), 124-132. 1, 1, 1

[28] B. G. Pachpatte, A note on Copson's inequality involving series of positive terms, Tamk. J. Math., 21 (1990), 13-19. 1

[29] S. H. Saker, Hardy-Leindler type inequalities on time scales, Appl. Math. Inf. Sci., 8 (2014), 2957-2981. 1

[30] S. H. Saker, D. O’Regan, R. P. Agarwal, Dynamic inequalities of Hardy and Copson types on time scales, Analysis., 34 (2014), 391-402. 1

[31] S. H. Saker, D. O’Regan, R. P. Agarwal, Generalized Hardy, Copson, Leindler and Bennett inequalities on time scales, Math. Nachr., 287 (2014), 686-698. 1

[32] S. H. Saker, R. R. Mahmoud, A. Peterson, Weighted inequalities of Hardy-type inequalities on time scales with applications, Mediterr. J. Math., 13 (2016), 585-606. 1, 2.2, 2.3

[33] S. H. Saker, R. R. Mahmoud, M. M. Osman, R. P. Agarwal, Some new generalized forms of Hardy's type inequality on time scales, Math. Inequal. Appl., 20 (2017), 459-481. 1, 1

[34] S. H. Saker, M. M. Abuelwafa, D. O'Regan, R. P. Agarwal, Norm dynamic inequalities and theorems of factorization of weighted Cesàro and Copson spaces, Bull. Braz. Math. Soc., (2019), 1-22. 2.4

[35] S. H. Saker, M. Kenawy, G. AlNemer, M. Zakarya, Some Fractional Dynamic Inequalities of Hardy's Type Via Conformable Calculus, Math., 8 (2020), 15 pages. 1

[36] H. Zhao, Z. Li, B. Li, T. Li, A study on input noise second-order filtering and smoothing of linear stochastic discrete systems with packet dropouts, Adv. Differ. Equ., 2020 (2020), 1-14. 1 